\title{
Performance Analysis of an Optoelectronic Localization System for Monitoring Brain Lesioning with Proton Beams
}

\author{
Fadi Shihadeh, Reinhard Schulte, Keith Schubert, and Pani Chakrapani
}

\begin{abstract}
Monitoring of the target position relative to the beam delivery system is a crucial requirement for creating small functional lesions in the brain with any radiosurgery modality. We have studied the performance of an optoelectronic localization system for monitoring brain lesioning with narrow proton beams. The system consists of three high-resolution cameras and dedicated software to locate a marker set in space. We tested the accuracy of the system by performing marker distance measurements and monitoring prescribed marker shifts with two different camera configurations and four different calibration techniques. Our results show that the camera-based alignment system appears adequate for the proposed task.
\end{abstract}

\section{INTRODUCTION}

Functional brain lesioning with stereotactic techniques has a long history and has been applied to the study of functional relationships in the animal brain and to treating functional brain disorders such as Parkinson's disease and trigeminal neuralgia. Lars Leksell, a Swedish neurosurgeon, was the first to suggest the use of multiple converging radiation beams for brain lesioning and coined the term "radiosurgery" for this technique [1]. This led to the development of the Gamma Knife, which is currently the most commonly used radiosurgical instrument for functional brain lesioning.

Radiosurgery is an attractive modality for brain lesioning because it is non-invasive except for the application of a stereotactic frame. A potential drawback is the lack for feedback provided by micro-electrode recording, which is important for some functional lesioning procedures such as pallidotomy [2]. This limitation may eventually be overcome with further development of functional imaging techniques such as functional MRI [3].

With increasing resolution of imaging techniques, brain lesioning with sub-millimeter accuracy becomes feasible. The overall application accuracy of current stereotactic radiosurgery systems, both frame-based and frame-less, is generally of the order of several millimeters [4] - [7] and has been traditionally limited by the resolution of the imaging modality used for targeting [8]. With steadily improving imaging resolution, other error sources become increasingly

This work was supported by the Henry L. Guenther Foundation

F. Shihadeh and Keith Schubert are with the Department of Computer Science, California State University, San Bernardino 5500 University Parkway, San Bernardino, CA 92407, USA fadi@r2labs.org, keithar2labs.org

R. Schulte is with the Department of Radiation Medicine, Loma Linda University Medical Center, 11234 Anderson Street, Loma Linda, CA 92354, USA rschulteddominion.llumc.edu

P. Chakrapani is with the Computer Science Program, 224 Appleton Hall, University of Redlands, Redlands, CA 92374, USA Pani_Chakrapani@redlands.edu important. Additional targeting errors can be introduced, for example, by a change in the patient position relative to the stereotactic reference system in the time interval between imaging and treatment [9].

Gamma knife and dedicated linear accelerators (linacs) with $0.5 \mathrm{~mm}$ or better isocentric accuracy are currently available technologies for functional lesioning in the brain. With the opening of the first hospital-based proton accelerator in Loma Linda, California in 1990, high energy protons delivered by isocentric proton gantries, which can rotate 360 degrees, are becoming an increasingly available modality for radiation therapy and radiosurgery [10]. Different from the other two modalities, protons can deliver both stopping beams with zero exit dose and sharp shoot-through beams, the latter being ideal for creating functional lesions.

Proton gantries, due to their large size and weight, are inherently less accurate in their isocenter definition than the compact gantries of modern photon linacs [11]. Therefore, for functional lesioning procedures additional equipment is required that tracks the alignment of the anatomical target to the beam axis with great accuracy. Over the last 20 years, navigational guidance systems have been developed for many applications including image-guided surgery (IGS) [12]. The registration technology, typically based on optical tracking of a rigid body of markers, and the related theory of errors in rigid body transformations [13], [14] are well applicable to tracking narrow proton beams relative to the target.

In this work, we report on the initial performance evaluation of a camera- and reflective-marker based optoelectronic localization system that we are planning to integrate into a positioning and alignment control system for functional proton radiosurgery at the Loma Linda University Medical Center proton treatment facility.

\section{MATERIALS AND METHODS}

\section{A. System Layout}

The hardware components of the optical positioning and alignment control system (OPACS) are shown in Fig. 1. Two retro-reflective marker sets are tracked by an optoelectronic localization system (OLS). The first set (target marker set) is attached to a stereotactic frame (Leksell G frame, Elekta Instruments, Stockholm, Sweden) and can display up to 17 markers. The target marker set represents the position of the anatomical target, as markers and target have known coordinates in the stereotactic reference system. The second set (beam marker set) includes 9 markers arranged as a cross and is attached to the proton beam applicator, a cone with 
a narrow collimator. It represents the position of the central proton beam axis within the proton gantry.

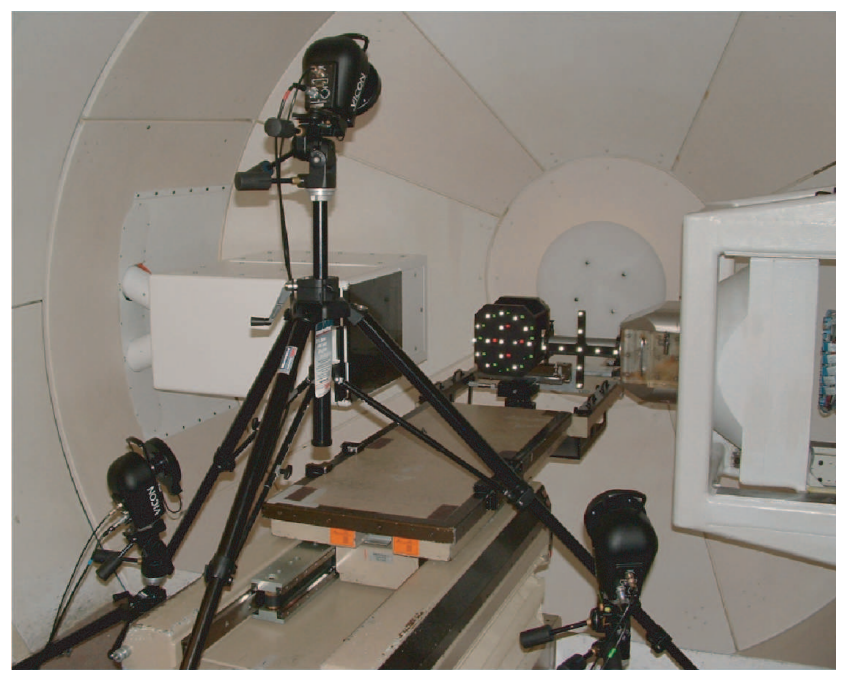

Fig. 1. Experimental setup with main hardware components of the OPACS.

Fig. 2 shows the principal operational scheme of the OPACS. The purpose of the OLS is to provide the 3D coordinates of the beam and target marker sets, which have known reference coordinates in their respective local coordinate systems tied to the beam applicator and stereotactic frame, respectively. The steps involved in the OLS operation are system calibration at the beginning of a measurement session, initial capture and definition of marker sets for automatic marker recognition, subsequent marker capture and conversion of marker images to single point coordinates, and transfer of marker coordinates to the alignment control system, a software algorithm and graphical user interface which determines and displays the actual offset between the beam axis and the target point. The offset vector is then transferred to the positioning system, which, in the final version, is planned to be a robotic patient positioner which performs the correction. The entire OPACS is a closed-loop circuit, which in case of a realtime OLS allows continuous monitoring of the target alignment relative to the beam axis.

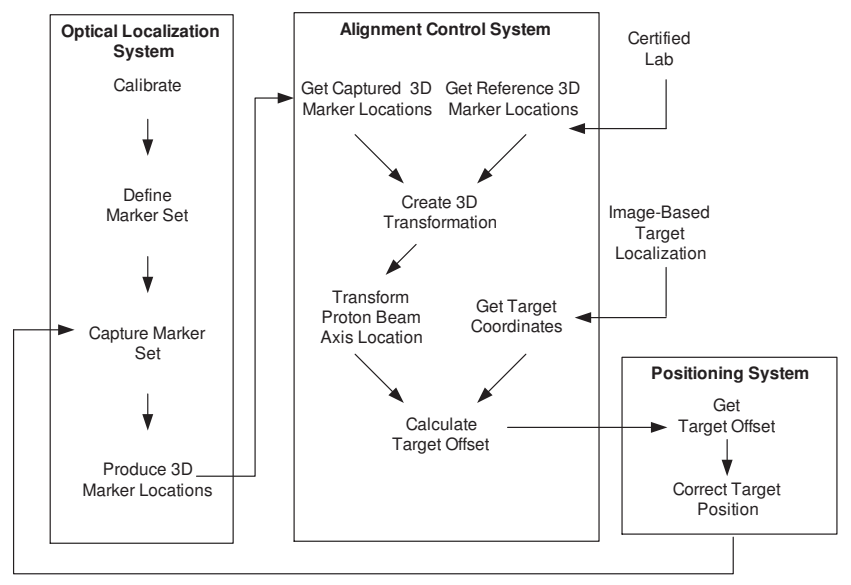

Fig. 2. Operational scheme of the OPACS.
In our initial studies, we have focused on the performance of the OLS. Knowledge of the coordinates of both marker sets in the global reference system of the OLS permits, in principle, calculation of the $3 \mathrm{D}$ vector translating the target point to the central beam axis. Accurate and reproducible measurement of the coordinates of the markers in a global reference system is a key requirement for the OLS. For the current study, the target marker set was used to study the performance accuracy of the OLS.

\section{B. Camera System}

For this study, the Vicon system (Vicon 260, Vicon Motion Systems, Ltd, Oxford, UK) was used as the OLS. The system comprised of three cameras (Mcam, 1 mega-pixel resolution, $12.5 \mathrm{~mm}$ focal length, $60 \mathrm{~Hz}$ ). For each data capture (trial), the system collected 600 frames per camera, which were read by Vicon's Workstation software on a dedicated workstation PC. The workstation software automatically recognizes a predefined marker set and calculates the 3D coordinates in the camera reference system established during system calibration (see next section). The coordinates of captured frames were averaged and read out into an ASCII text file by custom plugin software for further analysis.

\section{System Calibration}

At the beginning of each measurement session, a static and dynamic system calibration was performed utilizing Vicon's automatic calibration algorithm Dynacal3. The static calibration captured four spherical markers $(12 \mathrm{~mm})$ with L-shaped arrangement rigidly attached to a frame. A leastsquare best fit line through three horizontal markers established the horizontal (X) axis of the Vicon reference system. The vertical (Y) axis was defined as the line perpendicular to the first line passing through the remaining single marker, and the longitudinal (Z) axis was defined by the cross product of unit vectors in $\mathrm{X}$ and $\mathrm{Y}$ direction. One should note that for calibration the L-frame was inserted in the holder that normally holds the stereotactic frame, thus making the Vicon system and stereotactic system axes parallel.

The dynamic calibration was performed by waving a 100$\mathrm{mm}$ wand consisting of two spherical markers $(12 \mathrm{~mm})$ within the calibration volume, a cuboid of approximately $60 \mathrm{~cm}$ in side length. The exact distance between the centroids of the wand markers $(98.923 \mathrm{~mm})$, which is required for proper scaling, was measured by a certified inspection laboratory (Dimetrolab, Riverside, CA, USA). In addition, the distances between the individual markers of the L-frame were measured. These data were entered in the Calibration Reference Object (CRO) file of the Vicon software. In addition to the scaling factor, the dynamic calibration algorithm determined the position of the cameras relative to each other and the best-fit parameters of a linearization algorithm to correct for geometric lens distortions.

Each calibration produced three quality parameters: 1 . The camera residuals, defined as the rms difference between the reconstructed marker image, based on the data of two cameras and projected back to the image of the third camera, 
and the marker image measured by the third camera; 2 . the wand visibility, defined as the percentage of image frames with the wand seen by all three cameras; and 3 . the static reproducibility defined as the relative accuracy (in percent) with which the inspected distances between static Lframe markers (CRO file entries) were reproduced. Camera residuals of less than $1 \mathrm{~mm}$, wand visibility of $70 \%$ or better, and static reproducibilities of $1 \%$ or better were accepted as indicators for a suitable calibration.

\section{Camera Setup}

The three Vicon cameras were placed in a vertical equilateral triangle configuration and the camera plane was roughly parallel to the plane of the markers. Two individual camera configurations were tested within this scheme. The first configuration (see Fig. 1), called "standard configuration" as it can be conveniently realized at the back of the proton gantry enclosure, featured an equilateral triangle of 104 $\mathrm{cm}$ side length. The cameras were oriented such that their central axes met at a single point (isocenter) which was located central between the two marker sets. The central axes formed equal angles of about 50 degrees with respect to each other. The distance of the isocenter, from the camera plane was $110 \mathrm{~cm}$. The second camera configuration was an equilateral triangle with a side length of $177 \mathrm{~cm}$. The central axes intersected at an angle of 90 degrees at a distance of $70 \mathrm{~cm}$ from the camera plane. Again, the isocenter was placed at the center point between the two marker sets. We hypothesized that this "orthogonal configuration", although technically more difficult to realize, may lead to a higher degree of accuracy. For both camera configurations, the field of view of each camera at isocenter was $80 \mathrm{~cm}$, ensuring that both marker sets were included in the field of view of each camera and resolved with identical resolution.

\section{E. Experimental Design and Data Analysis}

The goal of this performance study was to characterize the systematic and random measurement errors of the OLS under realistic conditions and to identify the components in the variance of the error. The target marker set selected for this study consisted of 15 retro-reflective target markers (6 $\mathrm{mm}$ ) distributed over an area of about $20 \mathrm{~cm}^{2}$ in a plane parallel to the X-Y plane of the Vicon reference system.

The measurement accuracy and reproducibility of the system was studied using two endpoints: 1. the distances between each marker and the center of gravity (CG) of all other markers; and 2. the measured displacement of each marker after performing a prescribed shift in $\mathrm{X}, \mathrm{Y}$, or $\mathrm{Z}$ direction with micro-stages accurate to about $0.01 \mathrm{~mm}$. Distances between CG and marker location in the stereotactic reference system were known from dimensional inspection to $\pm 0.025 \mathrm{~mm}$. Differences between measured and nominal values were defined as distance and shift errors, respectively.

The performance study was organized into three experiments with camera configuration and calibration technique as the controlled experimental variables. The first experiment employed camera configuration 1 (standard) and a calibration technique with random wand movements. The experiment consisted of three sessions, with 18 individual data captures (trials). The trials included a start position and six prescribed moves per $X$ and $Z$ axis covering a range of $\pm 15 \mathrm{~mm}$ and five moves per $\mathrm{Y}$ axis covering a range from $-14 \mathrm{~mm}$ to $+7 \mathrm{~mm}$. Note that the $\mathrm{Y}$ axis had a more limited range of motion. The second experiment was identical in design but utilized camera configuration 2 (orthogonal).

The third experiment consisted of four sessions, each performed with camera configuration 1. For each session, a different calibration technique was used. The techniques differed with respect to the directionality of the wand movement: technique 1 used wand movement segments mostly perpendicular to the camera plane, technique 2 used movement segments mostly in vertical direction, and technique 3 used movement segments mostly in lateral direction; technique 4 , also used in the first two experiments, combined random movements in all directions. Each session included 17 trials with a reference position and 16 prescribed shifts divided among the three axes with a range similar to that in the first two experiments.

Distance errors and shift errors were analyzed with respect to normality of their distributions using KolmogorovSmirnov (KS) testing. For the first two experiments, grand means and standard deviations of marker session means were determined and compared using the Student t-test. For the third experiment, the marker session means and their standard deviations were compared with ANOVA. For each error type, variance and standard deviations were decomposed into marker, session, and trial effects using a linear statistical model of the form $y_{m s t}=\eta+\varepsilon_{m}+\varepsilon_{s}+\varepsilon_{t}$, where $y_{m s t}$ is the measurement of marker $m$ during trial $t$ of session $s, \eta$ is the population mean of all measurements, and $\varepsilon_{m}, \varepsilon_{s}$, and $\varepsilon_{t}$ are random variables that describe the effect of marker and inter- and intra-session variability on the measurement, respectively. An ANOVA table was constructed to derive an estimate for the standard deviation of each parameter in the model. The $95 \%$ confidence intervals of the standard deviations were derived by performing 1,000 or 10,000 simulations with the sample variances of the ANOVA table [15]. Interactions between marker, session and trial effects were not considered in this analysis.

\section{RESULTS}

\section{A. Calibration Factors}

We performed three experiments, the first two with three sessions for two different camera configurations using the same calibration technique, and the second with four sessions, one for each calibration technique using the same camera configuration. The three calibration parameters produced by the 10 sessions are summarized in Table I. This shows that the mean and maximum residual camera errors were well below $1 \mathrm{~mm}$. The visibility of the markers ranged from $68 \%$ to $98 \%$ and was typically above $90 \%$. The static reproducibility ranged from $0.48 \%$ to $1.08 \%$ and was typically below $1 \%$. No significant correlation between these 
TABLE I

CALIBRATION PARAMETERS

\begin{tabular}{llllll}
\hline Exp. & Session & $\begin{array}{l}\text { Camera } \\
(\text { Mean } \pm \text { SD) } \\
(\mathrm{mm})\end{array}$ & $\begin{array}{l}\text { Max } \\
\text { Res. } \\
(\mathrm{mm})\end{array}$ & $\begin{array}{l}\text { Visibility } \\
(\%)\end{array}$ & $\begin{array}{l}\text { Static } \\
\text { Reprod. } \\
(\%)\end{array}$ \\
\hline \multirow{3}{*}{1} & 1 & $0.43 \pm 0.07$ & 0.51 & 97 & 0.73 \\
& 2 & $0.47 \pm 0.10$ & 0.56 & 97 & 1.08 \\
& 3 & $0.48 \pm 0.03$ & 0.51 & 95 & 0.70 \\
\hline \multirow{3}{*}{2} & 1 & $0.37 \pm 0.07$ & 0.41 & 75 & 0.62 \\
& 2 & $0.26 \pm 0.02$ & 0.28 & 68 & 0.49 \\
& 3 & $0.70 \pm 0.11$ & 0.82 & 71 & 0.52 \\
\hline \multirow{3}{*}{3} & 1 & $0.53 \pm 0.04$ & 0.57 & 98 & 0.77 \\
& 2 & $0.54 \pm 0.07$ & 0.60 & 97 & 0.72 \\
& 3 & $0.45 \pm 0.06$ & 0.51 & 95 & 1.00 \\
& 4 & $0.44 \pm 0.05$ & 0.49 & 96 & 1.00 \\
\hline
\end{tabular}

factors and the session means of the distance and shift errors was found.

\section{B. Distance Error}

Distance errors were determined by calculating the difference between the distance of each marker from the CG of all remaining 14 markers and the corresponding distance measured by the dimensional metrology laboratory (DML).

During the first runs performed with the OLS, we noticed that the scaling factors determined by performing a linear regression of Vicon-measured CG distances against the DML CG distances, which ranged from $11.6 \mathrm{~mm}$ to $113.9 \mathrm{~mm}$, were slightly above or below 1.0 (typically 1-2\%). To make the absolute CG distance error independent of the magnitude of the CG distance, we henceforth determined the scaling factor for each measurement trial and used it to correct the CG distance accordingly. After this correction, no significant correlation was found between the error and the CG distance $(r=-0.15, p=0.60)$.

Exploration of the session data revealed no significant deviation of the distance error distribution from a normal distribution ( $p>0.05, \mathrm{KS}$ test). There was no significant correlation between the distance error and the size of the prescribed marker shift ( $r=0.44, p=0.32$ ), nor were the session means of the distance error different when grouped with respect to shift axes $\mathrm{X}, \mathrm{Y}$, or $\mathrm{Z}(p=0.62$, one-way ANOVA).

Table II shows a comparison of the mean distance errors for the standard and the orthogonal camera setup (experiment 1) and the four calibration techniques (experiment 2). These values represent systematic measurement errors. Tabulated means are the averages across sessions and markers and the standard deviations are for the marker means. All means were of the order of $0.1 \mathrm{~mm}$ with no significant differences between the two camera configurations and the four calibration techniques. Standard deviations of the marker means ranged from $0.155 \mathrm{~mm}$ to $0.213 \mathrm{~mm}$, representing the spread of systematic measurement errors between markers.

To study the variation of the individual measurement error, the standard deviations with $95 \%$ confidence intervals (in parentheses) of the distance measurement were obtained by ANOVA. These were $0.16 \mathrm{~mm}(0.12 \mathrm{~mm}, 0.25 \mathrm{~mm})$ for the
TABLE II

MEAN Distance ERRORS

\begin{tabular}{lclc}
\hline Parameter & Level & $\begin{array}{l}\text { Dist. Error } \\
(\mathrm{Mean} \pm \mathrm{SD})\end{array}$ & Probability $(\mathrm{p})$ \\
& & $\mathrm{mm})$ & \\
\hline Camera Setup & Standard & $0.088 \pm 0.155$ & 0.89 \\
& Orthogonal & $0.083 \pm 0.213$ & \\
\hline Calibration & 1 & $0.120 \pm 0.163$ & 0.71 \\
Technique & 2 & $0.094 \pm 0.166$ & \\
& 3 & $0.155 \pm 0.171$ & \\
& 4 & $0.160 \pm 0.179$ & \\
\hline
\end{tabular}

first experiment (camera configuration 1), $0.24 \mathrm{~mm}(0.19$ $\mathrm{mm}, 0.36 \mathrm{~mm}$ ) for the second experiment (camera configuration 2), and $0.17 \mathrm{~mm}(0.13 \mathrm{~mm}, 0.26 \mathrm{~mm})$ for the third experiment (four calibration techniques), demonstrating an unexpected, significantly larger random measurement error for the orthogonal camera configuration $(p<0.001$, F-test).

Fig. 3 shows the decomposition of the standard deviation with respect to marker, session, and trial (intra-session) effects for the three experiments. It illustrates that the betweenmarker variation of the error was the largest source of variation, followed by the inter-session variation, whereas the residual intra-session variation was relatively small (standard deviation less than $0.05 \mathrm{~mm}$ ). The inter-session standard deviation was significantly larger for camera configuration 2 , explaining the larger overall standard deviation of this experiment. The use of different camera calibration techniques for each session (experiment 3 ) did not significantly increase the inter-session variability compared to the other two experiments, which used only one calibration technique.

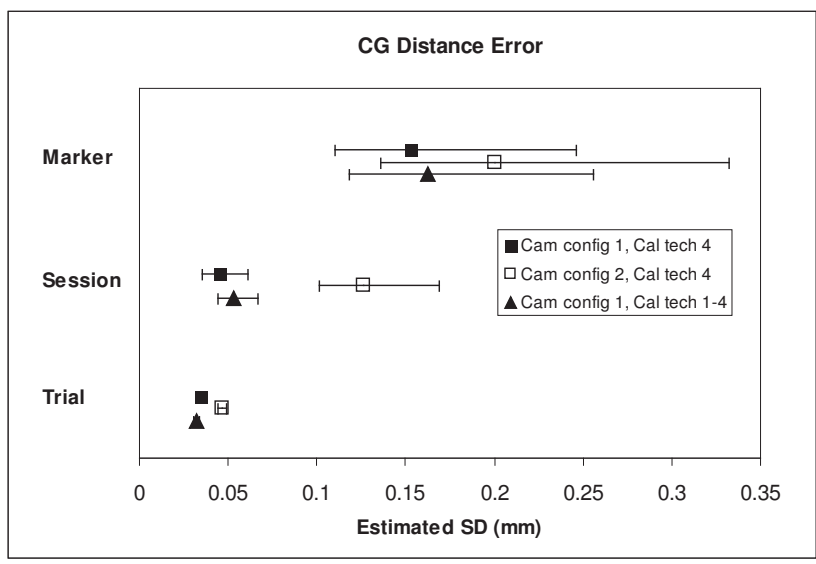

Fig. 3. Estimated standard deviation components and 95\% confidence intervals of the distance measurement.

\section{Shift Error}

Shift errors were determined by calculating the difference between prescribed shifts along the $\mathrm{X}, \mathrm{Y}$, or $\mathrm{Z}$ axis and measured shifts. For each session, shift measurements were corrected by the same scaling factor derived for CG distance data (see previous section). 
TABLE III

MEAN SHIFT ERRORS

\begin{tabular}{lclc}
\hline Parameter & Level & $\begin{array}{l}\text { Dist. Error } \\
(\mathrm{Mean} \pm \mathrm{SD})\end{array}$ & Probability (p) \\
& & $\mathrm{mm})$ & \\
\hline Camera Setup & Standard & $-0.036 \pm 0.018$ & 0.85 \\
& Orthogonal & $-0.035 \pm 0.018$ & \\
\hline Calibration & 1 & $-0.032 \pm 0.021$ & 0.075 \\
Technique & 2 & $-0.024 \pm 0.014$ & \\
& 3 & $-0.039 \pm 0.022$ & \\
& 4 & $-0.024 \pm 0.014$ & \\
\hline
\end{tabular}

Shift error distributions of individual sessions did not differ significantly from normal distributions $(p>0.05$, KS test). There was a weak correlation between the shift error and the size of the prescribed marker shift: Shift Error $(\mathrm{mm})=-0.0312+0.00452 *$ Shift $(\mathrm{mm}), r=0.521$, $p<0.0001$, probably due to residual scaling factor difference between measurements and micro-stage adjustments. Because the error introduced by this effect was very small, i.e., 4 micrometer per millimeter shift, no further correction to the shift error was made.

A small but significant difference of the mean shift error along the $\mathrm{Y}$ axis compared to the other two axes was found; the mean shift errors \pm standard errors for the $\mathrm{X}, \mathrm{Y}$,and $\mathrm{Z}$ axis were $-0.02 \mathrm{~mm} \pm 0.004 \mathrm{~mm},-0.07 \mathrm{~mm} \pm 0.004 \mathrm{~mm}$, and $0.02 \mathrm{~mm} \pm 0.004 \mathrm{~mm}$, respectively $(p<0.0001$, one-way ANOVA). This may be explained by the fact that the Y-axis micro-stage had to perform against the weight of stereotactic halo and target marker set.

Table III summarizes the mean shift errors and their standard deviations for the standard and the orthogonal camera setup (experiment 1) and the four calibration techniques (experiment 2). One should note that the mean shift errors were about three times and the standard deviations of the marker means about 10-times smaller than those for the distance errors.

The standard deviations with $95 \%$ confidence intervals (in parentheses) of the shift measurement were $0.09 \mathrm{~mm}$ $(0.086 \mathrm{~mm}, 0.095 \mathrm{~mm})$ for the first experiment (camera configuration 1), $0.089 \mathrm{~mm}(0.084 \mathrm{~mm}, 0.096 \mathrm{~mm})$ for the second experiment (camera configuration 2), and $0.100 \mathrm{~mm}$ $(0.096 \mathrm{~mm}, 0.104 \mathrm{~mm})$ for the third experiment (four calibration techniques), demonstrating no significant dependence on camera setup and calibration technique $(p>0.05$, Ftest). Fig. 4 shows the different components of the shift error standard deviation, illustrating that the contribution of variation between markers and sessions is very small in this case and practically all of the variation is due to intra-session variation. Also note that the latter is about two times larger than that of the distance error, which can be explained by the fact that the shift measurement consists of the difference between two marker coordinate measurements of about equal variance while the $\mathrm{CG}$ distance measurement involves the difference between a marker measurement and the average of 14 marker measurements (the CG location), which has a 14-times smaller variance then the individual marker measurement.

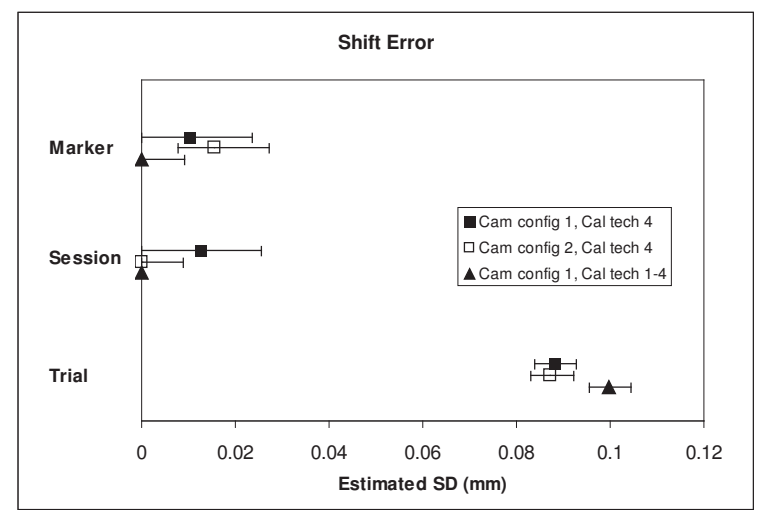

Fig. 4. Estimated standard deviation components and 95\% confidence intervals of the shift measurement. Negative variances were truncated.

\section{DISCUSSION AND CONCLUSION}

The goal of this investigation was to study the performance of an optoelectronic localization system for possible application in image-guided lesioning with narrow proton beams. With increasing capability for higher accuracy in animal and clinical brain lesioning procedures, due to better target localization, new techniques have to be developed that will allow application of sharp particle beams for this purpose.

Camera systems with active or passive markers attached to the human body are now widely used in image-guided surgery [16] as well as in image-guided radiotherapy and radiosurgery [17], [18]. The experience with these systems in the context of radiosurgery is, however, limited. After a review of available marker-based optical tracking systems, we selected the Vicon system, which is based on passive rather than active markers, due to its real-time capability of automatic marker registration and its use of high-resolution cameras. Wiles et al. [19] presented a general framework for characterization and calibration of optical guidance systems. They stressed that it is important to separate the accuracy obtained with a calibration procedure, and often stated by the manufacturer, from that obtained with an independent marker set, the latter being more important for a particular application. The authors also reported that passive markers provide a similar localization accuracy than active markers.

In this work, we studied two error endpoints, the distance error and the shift error, giving different information on the system performance. These errors belong to the category of fiducial localization errors (FLE) [13], [14]. Different from rms errors, which have a skewed, non-Gaussian distribution, the errors reported here have a Gaussian distribution around their means. They represent the measurement error components with respect to a given axis (shift error) or the line between a marker and the center of gravity of the remaining markers (distance error) rather than the absolute values of the error vectors. Since we found no directionality in these errors, they can be used to derive the rms localization errors for the purpose of comparison with other systems. 
The distance error is important for the accuracy and precision of localizing the target and beam markers with respect to each other. Systematic and random system errors were of the order of 0.1 and $0.2 \mathrm{~mm}$, respectively, which is acceptable for the purpose of sub-millimetric alignment accuracy. We found that a significant source of the overall variation as well as the systematic error was due to the markers themselves. This is probably related to the fact that the markers are made of a spherical ceramic core wrapped with retro-reflective tape, which introduces some variation in their spherical symmetry. A careful marker selection could potentially improve the OLS accuracy and reproducibility to better than $0.1 \mathrm{~mm}$.

We found that the orthogonal camera configuration had a significantly larger distance measurement uncertainty than the standard camera setup with about 50 degrees between the central camera axes. This unexpected result may be explained by the fact that the Vicon markers are incomplete spheres due to a flat part serving for marker attachment to their posts. With the standard camera arrangement, this part was practically invisible to the cameras, while for the orthogonal arrangement it was partially visible.

The second study endpoint, the shift error, is a measure of the accuracy and precision of spatial shifts with respect to a reference position. This is important for realtime tracking of small motions of the target and beam relative to each other. Overall, this error was about one magnitude smaller than the distance error. This can be attributed to the fact that this error is rather independent of the marker quality since the shift measurement only tracks relative changes in the position of the same markers while the distance measurement involves the position of each marker relative to all other markers. Thus if the marker is perceived in the wrong location, this will affect its distance but not its shift measurement.

In addition to marker variability, both intra- and intersession variability contribute to both distance and shift measurement errors. The intra-session error is probably due to random internal error sources, such as electronic noise and marker flickering. The inter-session error could be related to the variability in the manually calibrated measurement volume of the system. The intra-session error may be reduced with technical advances in marker recognition and low-noise electronics, or by adding additional cameras and decreasing the distance between cameras and markers. The inter-session error may be improved by standardizing the dynamic calibration technique with a robotic system.

It should be stressed that our project is work in progress. In addition to the fiducial localization error, the target registration error (TRE) needs to be addressed. The geometrical configuration of the fiducial markers and their distance from the anatomical target influence the application accuracy of rigid-transformation based guidance systems [19], [20]. Further, one needs to address the stability of the patient position relative to the stereotactic reference frame [9].

In conclusion, this initial OLS performance study has shown that the Vicon system model 260 in combination with passive retro-reflective markers appears adequate for the stated purpose monitoring functional proton lesioning procedures with sub-millimeter accuracy. The application accuracy of the integrated OPACS, which depends on many additional factors, has yet to be tested.

\section{ACKNOWLEDGMENTS}

The authors would like to thank Jongmin Cho, Ying Nie, and Kevin Webster for their assistance with the experimental setup. Dr. Khaled Bahri is thanked for his valuable assistance with the statistical analysis.

\section{REFERENCES}

[1] L. Leksell, The stereotaxic method and radiosurgery of the brain, Acta Chir. Scand., vol. 102, 1951, pp 316-319.

[2] K. Tsao, S. Wilkinson, J. Overman et al. Pallidotomy lesion locations: significance of microelectrode refinement, Neurosurgery vol. 43, 1998, pp 506-512;

[3] R. Bammer, S. Skare, R. Newbould, et al., Foundations of advanced magnetic resonance imaging, NeuroRx. vol. 2, 2005, pp 167-196.

[4] R. J. Maciunas, R.L. Galloway Jr., and J.W. Latimer, The application accuracy of stereotactic frames, Neurosurgery vol. 35, 1994 pp 682-94.

[5] S.D. Chang, W. Main, D.P. Martin et al., An analysis of the accuracy of the CyberKnife: a robotic frameless stereotactic radiosurgical system, Neurosurgery vol. 52, 2003, pp 140-146.

[6] J.M. Henderson, Frameless localization for functional neurosurgical procedures: a preliminary accuracy study, Stereotact. Funct. Neurosurg. vol. 82, 2004, pp 135-141.

[7] A. Quinones-Hinojosa, M.L. Ware, N. Sanai and M.W. McDermott, Assessment of image guided accuracy in a skull model: comparison of frameless stereotaxy techniques vs. frame-based localization. $J$. Neurooncol. vol. 76, 2006, pp 65-70.

[8] W. Zylka and J. Sabczynski, Effect of localization devices and registration methods on the accuracy of stereotactic frame systems predicted by the Gaussian approach, Comput. Aided Surg. vol. 4, 1999, pp 77-86.

[9] Torsten Rohlfing, Calvin R. Maurer, Jr., David Dean, and Robert J. Maciunas. Effect of changing patient position from supine to prone on the accuracy of a Brown-Roberts-Wells stereotactic head frame system. Neurosurgery, 52(3):610-618, 2003.

[10] D. Schulz-Ertner, O. Jakel, and W. Schlegel, Radiation therapy with charged particles. Semin. Radiat. Oncol. vol. 16, 2006, pp 249-59.

[11] M.F. Moyers and W. Lesyna, Isocenter characteristics of an external ring proton gantry. Int. J. Radiat. Oncol. Biol. Phys. vol. 60, 2004, pp 1622-1630.

[12] Greg Welch and Eric Foxlin. Motion tracking: No silver bullet, but a respectable arsenal. IEEE Comput. Graph. Appl., 22(6):24-38, 2002.

[13] J. Michael Fitzpatrick, Jay Bailey West, and Clavin R. Maurer, Jr. Predicting error in rigid-body, point-based registration. IEEE Trans. Med. Imag, 17(5):694-702, 1998.

[14] J. Michael Fitzpatrick and Jay B. West. The distribution of target registration error in rigid-body, point-based registration. IEEE Trans. Med. Imag, 20(9):917-927, 2001.

[15] A. Gelman, Analysis of variance - why is it more important than ever. Ann. Statist. vol. 33, 2005, pp 1-53.

[16] Terry M. Peters. Image-guidance for surgical procedures. Phys. Med. Biol., 51(14):R505-R540, 2006.

[17] S.L. Meeks, F.J. Bova, W.A. Friedman et al., IRLED-based patient localization for linac radiosurgery. Int. J. Radiat. Oncol. Biol. Phys. vol. 41, 1998 pp 433-439.

[18] W.A. Tome, S.L. Meeks, J.M. Buatti et al., A high-precision system for conformal intracranial radiotherapy. Int. J. Radiat. Oncol. Biol. Phys. vol. 47, 2000 pp 1137-1143.

[19] Andrew D. Wiles, David G. Thompson, and Donald D. Frantz. Accuracy assessment and interpretation for optical tracking systems. In SPIE Medical Imaging: Visualization, Image-Guided Procedures, and Display, pages 421-432, 2004.

[20] Jay B. West and Calvin R. Maurer, Jr. Designing optically tracked instruments for image-guided surgery. IEEE Trans. Med. Imag, 23(5):533-545, 2004. 